\title{
Article
}

\section{MiR-208b Regulates Rabbit Preadipocyte Proliferation and Differentiation}

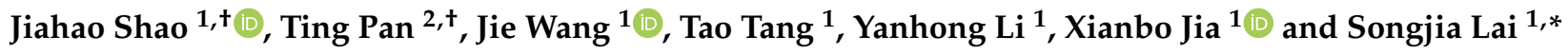 \\ 1 College of Animal Science and Technology, Sichuan Agricultural University, Chengdu 611130, China; \\ shaojh1997@126.com (J.S.); wjie68@163.com (J.W.); m18483220592@163.com (T.T.); \\ lyh81236718@163.com (Y.L.); jaxb369@sicau.edu.cn (X.J.) \\ 2 College of Veterinary Medicine, Sichuan Agricultural University, Chengdu 611130, China; \\ panting555666@163.com \\ * Correspondence: laisj5794@163.com \\ + Jiahao Shao and Ting Pan contributed equally to this work.
}

Citation: Shao, J.; Pan, T.; Wang, J.;

Tang, T.; Li, Y.; Jia, X.; Lai, S.

MiR-208b Regulates Rabbit

Preadipocyte Proliferation and

Differentiation. Genes 2021, 12, 890.

https://doi.org/10.3390/

genes12060890

Academic Editor: Eric Barrey

Received: 18 March 2021

Accepted: 3 June 2021

Published: 9 June 2021

Publisher's Note: MDPI stays neutral with regard to jurisdictional claims in published maps and institutional affiliations.

Copyright: (c) 2021 by the authors. Licensee MDPI, Basel, Switzerland. This article is an open access article distributed under the terms and conditions of the Creative Commons Attribution (CC BY) license (https:/ / creativecommons.org/licenses/by/ $4.0 /)$.

\begin{abstract}
RNAs (miRNAs) play an important role in gene regulation in animals by pairing with target gene mRNA. Many miRNAs are differentially expressed in the adipose tissue, often with conserved expression. In our study, we found that miR-208b expression was observed differently in the preadipocyte differentiation model. When miR-208b was overexpressed in the preadipocyte differentiation model, the overexpressed group displayed higher expression of PPAR $\gamma$ and FABP4 - the markers of preadipocyte differentiation. Oil Red O staining revealed that the count of lipid droplets was increased in the overexpressed group. When the expression of miR-208b was inhibited, the above indicators showed an opposite trend. Moreover, results from both 5-ethynyl-2'-deoxyuridine (EDU) and cell counting kit (CCK) analysis showed that miR-208b promoted the proliferation of preadipocyte. Expression of gene CSNK2A2, a direct miR-208b target, was downregulated in the overexpressed group, providing a possible link to multiple signal pathways. Overall, our data indicate that miR-208b play a positive regulatory effect on the proliferation and differentiation of rabbit preadipocyte.
\end{abstract}

Keywords: microRNAs; preadipocyte; proliferation; differentiation

\section{Introduction}

During the past five decades, the prevalence of obesity has rapidly increased, becoming a serious public health issue [1]. Obesity is characterized by the excess accumulation of adipose tissue under the definition of the World Health Organization (WHO) [2]. Overall, adipogenesis is a complex process that is associated with the dynamic expression of multiple transcription factors [3]. Proliferation and differentiation of preadipocyte induce the number of mature adipocyte capable of triglyceride storage, metabolism, and production of adipokines [4]. Rabbit is an ideal experimental animal to study adipogenesis due to its lipid metabolism and obesity-related clinical manifestations similar to those of humans $[5,6]$. Moreover, compared with other meat, rabbit meat has high digestibility, which is not available in other meat. The rabbit industry plays an important role in the national economy, especially in developing countries [7]. Extensive evidence suggests that meat quality and meat flavor have a great relationship with the fat content of animals [8,9]. Thus, a detailed study of the regulatory mechanisms that underlie rabbit preadipocyte proliferation and differentiation would be expected to contribute to the understanding of adipogenesis and rabbit breeding.

miRNAs have been validated to be able to pair with the $3^{\prime}$ untranslated region (UTR) of target gene mRNA and regulate the target gene at the post-transcriptional level [10]. Extensive work revealed that miRNAs are involved in the regulation of preadipocyte proliferation and differentiation. For example, a previous study reported that miR-143a$3 p$ modulates preadipocyte proliferation and differentiation by targeting MAPK7 [11]. 
miR-125a-5p promotes 3T3-L1 preadipocyte proliferation, while negatively regulating STAT3 to inhibit 3T3-L1 preadipocyte differentiation [12]. Previous studies have found that miR-208b is a key molecule that poses the regulated function for a variety of diseases including chronic chagas disease cardiomyopathy and acute myocardial infarction [13,14]. Moreover, it is important that miR-208b regulates intracellular fatty acid and glucose metabolism and influences the development of obesity [15]. The above studies revealed that miR-208b may regulate the proliferation and differentiation of preadipocyte, but the specific mechanism remains unclear. Therefore, we investigated the role of miR-208b in rabbit preadipocyte proliferation and differentiation by overexpressing or inhibiting miR-208b.

\section{Materials and Methods}

\subsection{Animals and Cell Collection}

A newborn New Zealand rabbit was used for sampling. Briefly, the experimental rabbit was rubbed all over with alcohol and sacrificed humanely to reduce suffering. The primary preadipocyte was collected in the perirenal area of newborn New Zealand rabbits using the method described earlier [16]. All experiments in the present trial involving animals were performed under the direction of the Institutional Animal Care and Use Committee from the College of Animal Science and Technology, Sichuan Agricultural University, China (DKY-B2019202015).

\subsection{Cell Culture}

Preadipocyte was maintained in a growth medium (GM) containing $10 \%$ fetal bovine serum (FBS, Gibco, Carlsbad, CA, USA) in an incubator (Thermo Scientific, Waltham, MA, USA) at $37^{\circ} \mathrm{C}$ and $5 \% \mathrm{CO}_{2}$ after sterility separation, and the medium was changed every two days. When the cell density reached $80 \%$, it was purified and passage to obtain the third generation of preadipocyte for subsequent experiments. To stimulate preadipocyte differentiation, the third generation of preadipocyte was cultured in differentiation media (DM) containing Dulbecco's modified Eagle's medium (DMEM, Gibco, Carlsbad, CA, USA) supplemented with 5\% FBS, $0.5 \mathrm{mM}$ dexamethasone (DEX), $0.5 \mathrm{mM}$ 3-isobutyl-1methylxanthine (IBMX), and $10 \mathrm{mg} / \mathrm{mL}$ insulin for three days. After that, the medium was replaced by a maintenance medium (MM) consisting of DMEM with 5\% FBS and $10 \mathrm{mg} / \mathrm{mL}$ insulin for three days. Subsequently, the medium was replaced with GM containing $5 \%$ FBS.

\subsection{Transfection}

When the cell density reached approximately $80 \%$ confluence, they were transfected with miR-208b mimic, miR-208b inhibitor, miR-208b negative control (NC), and miR-208b inhibitor negative control (INC) using the lipofectamine 3000 reagent (Invitrogen, Carlsbad, CA, USA), following the manufacturer's protocol. The above RNA oligo (mimic, inhibitor, NC, INC) was purchased from the Sangon Bioengineering Co., Ltd (Shanghai, China), and the sequences are shown in Table 1. Subsequently, the cell was kept in the transfection reagent for $6 \mathrm{~h}$ and then changed to the corresponding DM to induce differentiation. For cell proliferation experiments, transfection was performed as described above when the cell density reached $50 \%$. After $6 \mathrm{~h}$, the medium was changed to GM.

Table 1. The sequence information of RNA oligo.

\begin{tabular}{cc}
\hline Name & Sequence Information $\left(\mathbf{5}^{\prime}-\mathbf{3}^{\prime}\right)$ \\
\hline miR-208b mimic & F:AUAAGACGAACAAAAGGUUUGU \\
miR-208b inhibitor & R:ACAAACCUUUUGUUCGUCUUAU \\
miR-208b NC & ACAAACCUUUUGUUCGUCUUAU \\
miR-208b INC & F:UUGUACUACACAAAAGUACUG \\
& R:GUACUUUUGUGUAGUACAAUU \\
\hline
\end{tabular}




\subsection{Quantitative Real-Time Polymerase Chain Reaction Analysis}

Total RNA from the cell was obtained using the RNAiso Plus Reagent (Invitrogen, Hong Kong, China) according to the standard guidelines. The Agilent 2100 Bioanalyzer system (Agilent Technologies, Carlsbad, CA, USA) was used for quality inspection, and only qualified RNA (A160/A180 $=1.6-1.8$, concentration $>200 \mathrm{ng} / \mathrm{uL}$ ) was used for the later trial. Reverse transcription of mRNA and miRNA was performed using the PrimeScript $^{\mathrm{TM}}$ RT reagent Kit with gDNA Eraser (Takara, Dalian, China) and the Mir$\mathrm{X}^{\mathrm{TM}}$ miRNA First-Strand Synthesis Kit (Takara) following the manufacturer's protocol, respectively. Subsequently, qRT-PCR was performed in triplicate using the TB Green ${ }^{\mathrm{TM}}$ Premix Ex Taq ${ }^{\mathrm{TM}}$ II (Takara) on a CFX96 instrument (Bio-Rad, Hercules, CA, USA), and the relative expression levels of mRNA and miRNA were calculated using the $2^{-\Delta \Delta \mathrm{Ct}}$ method. The mRQ $3^{\prime}$ primer in the Mir- $X^{\mathrm{TM}}$ miRNA First-Strand Synthesis Kit (Takara) was used and served as a reverse primer for miRNA quantification, and U6 was used as an internal reference. Besides, GAPHD was used as an internal reference for mRNA quantification. Detailly, the primer sequences are listed in Table 2.

Table 2. The primer sequences were used for the qRT-PCR analysis.

\begin{tabular}{ccc}
\hline Name & Forward Primer $\left(\mathbf{5}^{\prime} \mathbf{- 3}^{\prime} \mathbf{)}\right.$ & Reverse Primer $\left(\mathbf{5}^{\prime} \mathbf{- 3}^{\prime} \mathbf{)}\right.$ \\
\hline$P P A R \gamma$ & GAGGACATCCAGGACAACC & GTCCGTCTCCGTCTTCTTT \\
FABP4 & GGCCAGGAATTTGATGAAGTC & AGTTTATCGCCCTCCCGTT \\
GAPDH & CTTCGGCATTGTGGAGGG & GGAGGCAGGGATGATGTTCT \\
CSNK2A2 & GTGCTCTCCAGTGGTCTCAC & GGACAACAGGAACCGACCAT \\
miR-208b & ATAAGACGAACAAAAGGTTTGT & mRQ 3' primer \\
U6 & GGAACGATACAGAGAAGATTAGC & TGGAACGCTTCACGAATTTGCG \\
\hline
\end{tabular}

\subsection{Protein Extraction and Western Blotting}

Total protein from the cell was collected using a commercial Protein Extraction Kit (Sangon, Shanghai, China), following the manufacturer's protocol. The concentration of the protein was measured using the Bradford protein assay kit (Beyotime, Jiangsu, China), and only protein meeting quality criteria were used for the further trial. Briefly, the protein was resolved on 10\% SDS-PAGE and then transferred to a PVDF membrane, followed by sealing of the sealing fluid. The membranes were incubated with the correspondingly primary antibodies for $24 \mathrm{~h}$ at $4{ }^{\circ} \mathrm{C}$ and subsequently incubated with the secondary antibodies for $2 \mathrm{~h}$. The membranes were subjected to chemiluminescence reagents to detect immunoreactivities. A GelDoc system equipped (Bio-Rad, Hercules, CA, USA) was used to capture images. $\beta$-actin protein was used as an internal control.

\subsection{CCK Assay}

Cell counting kit (CCK, GOYOD, Nanjing, China) was used to examine the effect of $\mathrm{miR}-208 \mathrm{~b}$ on preadipocyte proliferation. Briefly, the third generation of preadipocyte was seeded in 96-well plates and transfected with miR-208b mimic, inhibitor, NC, and INC at $50 \%$ cell density. Six hours later, the medium was changed to GM. After incubation for $24 \mathrm{~h}, 48 \mathrm{~h}, 72 \mathrm{~h}, 96 \mathrm{~h}$, and $120 \mathrm{~h}, 10 \mu \mathrm{L}$ of CCK reagent was added to each well and incubated at $37{ }^{\circ} \mathrm{C}$ and $5 \% \mathrm{CO}_{2}$ for $2 \mathrm{~h}$. In living cells, 2-(2-methoxy-4-nitrophenyl)3-(4-nitrophenyl)-5-(2,4-disulfophenyl)-2h-tetrazolium sodium salt is reduced to orange formazan by dehydrogenase. The number of living cells is proportional to the amount of formazan, the amount of formazan is proportional to the absorbance at $450 \mathrm{~nm}$. Thus, the absorbance of the sample can represent the number of living cells and was measured by a microplate reader (Thermo Scientific, Waltham, MA, USA).

\subsection{EDU Proliferation Assay}

Preadipocyte was grown in 12-well plates. Transfection and medium replacement were performed as described above. After $48 \mathrm{~h}$, the cell was cultured for $2 \mathrm{~h}$ in GM containing $100 \mu \mathrm{L}$ 5-ethynyl-2'-deoxyuridine (EDU, RiboBio, Guangzhou, China). Next, 
the cell was immobilized and stained according to the company's instructions. The staining of EDU and Hoechst in the same field were photographed using an inverted fluorescence microscope (Olympus, Tokyo, Japan). Images were analyzed by using image-pro plus 6.0 software (Media Cybernetics, Inc, Rockville, MD, USA).

\subsection{Oil Red O Staining}

Oil Red O staining was performed at room temperature. The cell was washed three times with phosphate-buffered saline (PBS) and fixed in 10\% paraformaldehyde for $30 \mathrm{~min}$. Subsequently, Oil Red O was mixed with deionized water at a rate of 3:2 and then added to the stained cell for $1 \mathrm{~h}$. Finally, the above cell was rinsed with PBS until there were no obvious impurities. Images were captured using an inverted microscope (Olympus). Moreover, the count of lipid droplets was measured by using image-pro plus 6.0 software (Media Cybernetics).

\subsection{Target Genes Prediction and Verification}

The target genes of miR-208b were predicted using the online database TargetScan (http:/ / www.targetscan.org/mamm_31/). Gene Ontology (GO) analysis and Kyoto Encyclopedia of Genes and Genomes (KEGG) pathway enrichment analysis were performed using software DAVID 6.7 (http:/ / david.abcc.ncifcrf.gov/home.jsp). Moreover, the MiRWalk database (http:/ / zmf.umm.uni-heidelberg.de/apps/zmf/mirwalk2/) was used to predict the potential site that binds with miR-208b. To validate the binding site, luciferase reporter plasmids (wild-type and mutant $3^{\prime}$ UTR of CSNK2A2) were constructed by Tsingke Biotechnology Co., LTD (Tsingke, Chengdu, China). Hela cell was seeded into 24-well plates. The wild-type or mutant plasmids were cotransfected with miR-208b mimic into Hela cell when the cell density reached 70\%. Then, luciferase activities were measured using the TransDetect ${ }^{\circledR}$ Double Luciferase Reporter Assay Kit instructions (Transgen, Beijing, China) after $24 \mathrm{~h}$, following the manufacturer's guides.

\subsection{Statistical Analysis}

All data are presented as means \pm SEM. The SPSS 22.0 software (SPSS Inc. Chicago, IL, USA) was used for statistical analysis, and differences between groups were determined by Student's $t$-test. Moreover, multiple comparisons were performed using one-way ANOVA followed by Dunnett's posthoc analysis. Two-way ANOVA was performed to compare differences between the two groups for PPAR $\gamma$ and FABP4. Differences were considered statistically significant at $p<0.05$.

\section{Results}

\subsection{Establishment of Rabbit Preadipocyte Differentiation Model}

To establish a model of rabbit preadipocyte differentiation, the third generation of preadipocyte was cultured in cell culture plates and differentiated with the aforementioned method when the density reached $80 \%$. Results of Oil Red O staining showed that the number of lipid droplets was significantly increased during the differentiation progress (Figure 1a,b). Simultaneously, preadipocyte differentiation key genes PPAR $\gamma$ and FABP4 expression were significantly upregulated in the differentiation model and had the highest levels on day 4 (Figure 1c,d). Therefore, we conclude that the preadipocyte was successfully induced to differentiate. Besides, miR-208b expression was markedly different expression in the adipocyte differentiation model, revealing a possible regulatory role of miR-208b in preadipocyte differentiation (Figure 1e). 


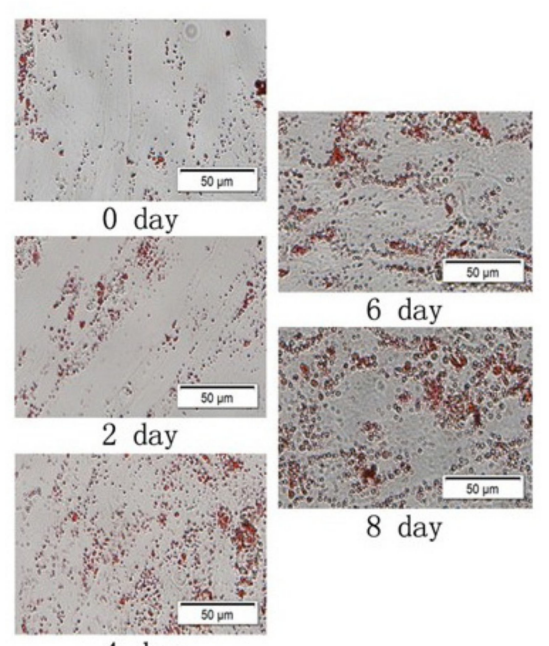

(a)

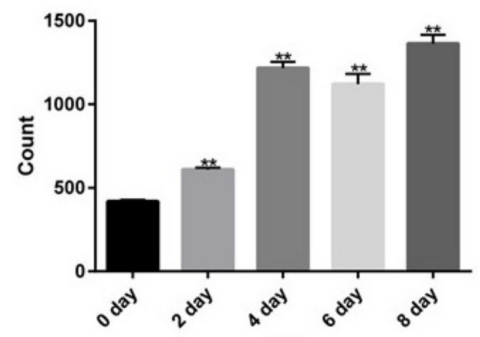

(b)

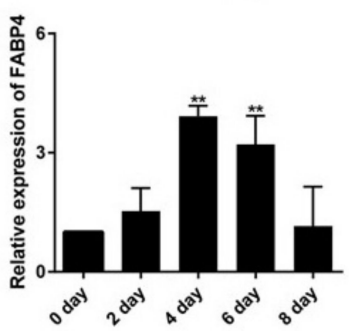

(d)

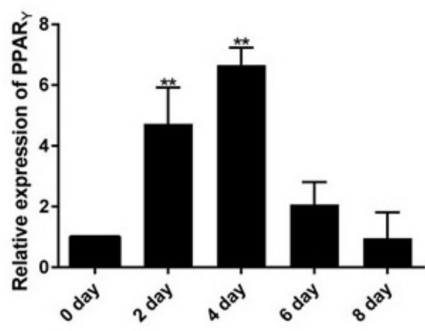

(c)

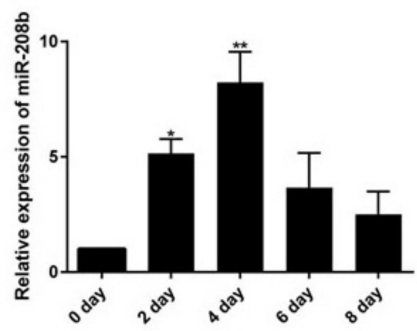

(e)

Figure 1. Establishment of rabbit preadipocyte differentiation model. (a) Oil Red O staining of lipid droplets at 0,2, 4, 6, and 8 days of differentiation. (b) The number of lipid droplets $(n=3)$. (c,d) The relative expression levels of PPAR $\gamma$ and FABP4 in rabbit preadipocyte after inducing differentiation at $0,2,4,6$, and 8 days. (e) The relative expression levels of miR-208b during preadipocyte differentiation progress. The data are presented as means \pm SEM $(n=9) .{ }^{*} p<0.05 ;{ }^{* *} p<0.01$.

\subsection{MiR-208b Promotes Rabbit Preadipocyte Differentiation}

To assess the functional effect of miR-208b on rabbit preadipocyte differentiation, we transfected the miR-208b mimic, miR-208b inhibitor, NC, and INC into the preadipocyte, which was differentiated with the aforementioned method. As shown in Figure 2a. The relative expression levels of miR-208b in the mimic (inhibitor) group were significantly higher (lower) than those in the NC (INC) group, indicating that transfection analogs successfully increased (decreased) the expression levels of miR-208b. Oil Red O staining showed that the number of lipid droplets was higher in the mimic group than the NC group, but the number of lipid droplets was lower in the inhibitor group than the INC group after 8 days of transfection (Figure $2 \mathrm{~b}, \mathrm{c}$ ). Furthermore, we found an obvious and highly significant increase of genes in the mimic group, including PPAR $\gamma$ and FABP4, which are important transcription factors in the development and function of the adipose tissue and markers of preadipocyte differentiation (Figure 2d,e). In contrast, $P P A R \gamma$ and FABP4 were expressed at lower levels in the inhibitor group (Figure 2f,g). We validated the qRT-PCR data by WB assays, using the total protein from the miR-208b mimic, miR-208b inhibitor, NC, and INC groups. We confirmed an increased expression of PPAR $\gamma$ and FABP4 at the protein levels in the mimic group, but a decreased expression of PPAR $\gamma$ and FABP4 in the inhibitor group (Figure $2 \mathrm{~h}$ ). Therefore, we concluded that miR-208b play a positive role in rabbit preadipocyte differentiation. 


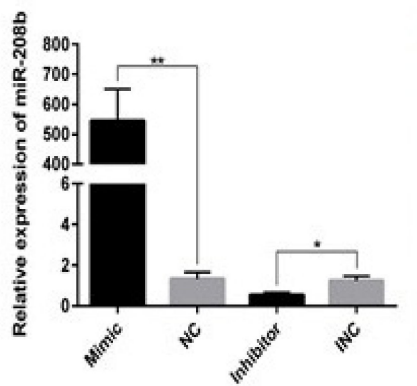

(a)

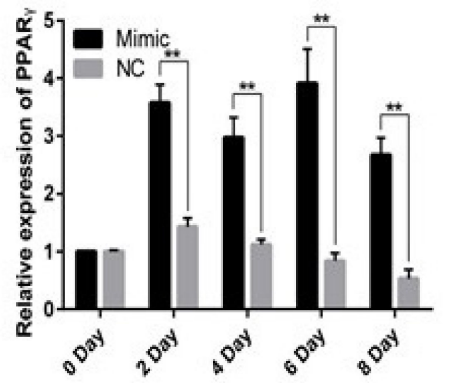

(d)

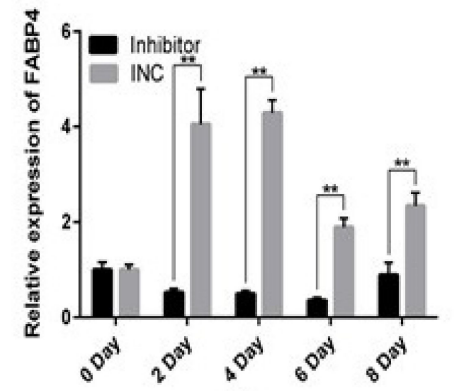

(g)

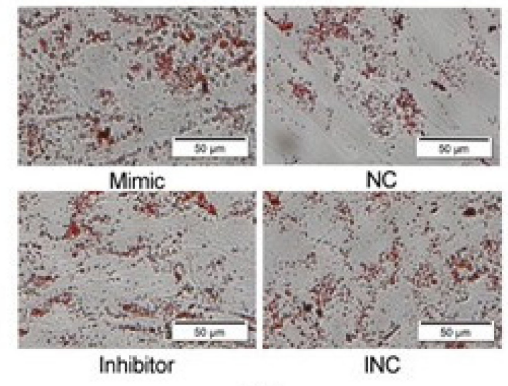

(b)

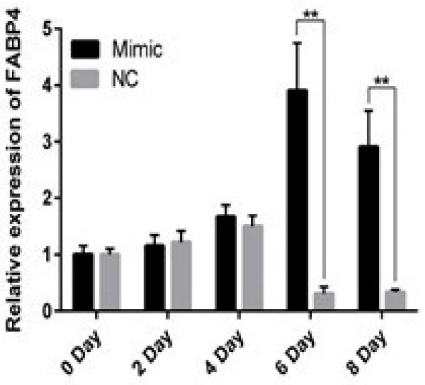

(e)

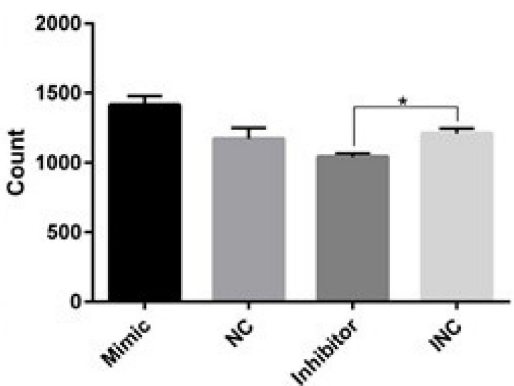

(c)

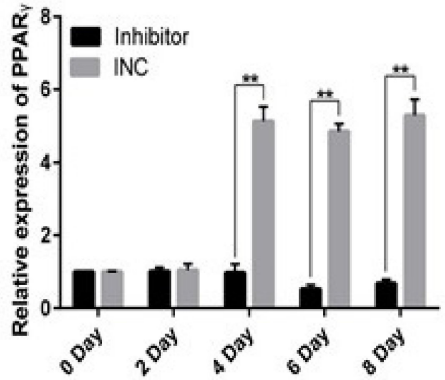

(f)

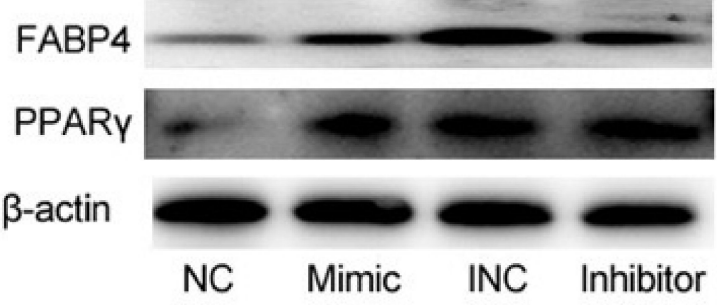

(h)

Figure 2. miR-208b promotes rabbit preadipocyte differentiation. (a) Transfection efficiency detection of miR-208b mimic and miR-208b inhibitor. (b) Oil Red O staining of lipid droplets after 8 days of transfection. (c) The number of lipid droplets $(n=3)$ after 8 days of transfection. (d,e) The relative expression levels of PPAR $\gamma$ and FABP 4 in rabbit preadipocyte induced differentiation at $0,2,4,6$, and 8 days after transfecting with miR-208b mimic and NC. (f,g) The relative expression levels of $P P A R \gamma$ and $F A B P 4$ in rabbit preadipocyte induced differentiation at $0,2,4,6$, and 8 days after transfecting with miR-208b inhibitor and INC. (h) $P P A R \gamma$ and FABP4 protein levels during preadipocyte differentiation after transfecting with NC, miR-208b mimic, INC, and miR-208b inhibitor. The data are presented as means $\pm \operatorname{SEM}(n=9) .{ }^{*} p<0.05 ;{ }^{* *} p<0.01$.

\subsection{MiR-208b Promotes Rabbit Preadipocyte Proliferation}

We performed CCK and EDU proliferation assay by transfecting the miR-208b mimic, miR-208b inhibitor, NC, and INC into the preadipocyte cultured in vitro to assess the effect of miR-208b on rabbit preadipocyte proliferation. As shown in Figure 3a,b, the miR-208b mimic significantly increased the absorbance of preadipocyte after incubation for $24 \mathrm{~h}$ $(p<0.05), 48 \mathrm{~h}(p<0.01), 72 \mathrm{~h}(p<0.001), 96 \mathrm{~h}(p<0.001)$, and $120 \mathrm{~h}(p<0.05)$, but the miR$208 \mathrm{~b}$ inhibitor significantly decreased the absorbance of the preadipocyte after incubation for $48 \mathrm{~h}(p<0.05), 72 \mathrm{~h}(p<0.05), 96 \mathrm{~h}(p<0.001)$, and $120 \mathrm{~h}(p<0.05)$. Besides, the EDU proliferation assay revealed that the number of positive cells was significantly higher in the mimic and INC groups than the NC and inhibitor groups, respectively (Figure 3c,d). These data suggest that miR-208b play a positive role on rabbit preadipocyte proliferation. 


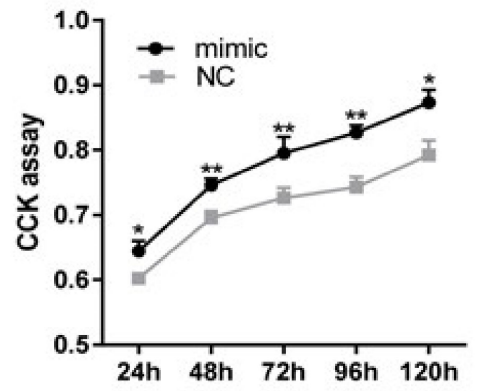

(a)

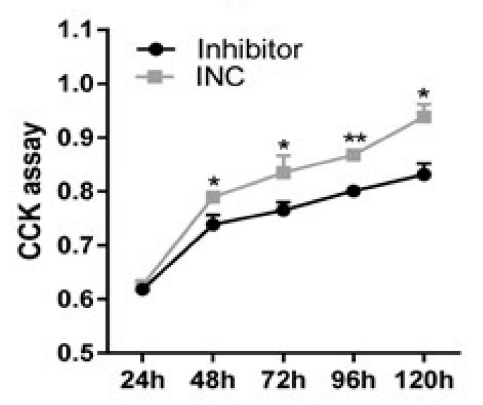

(b)

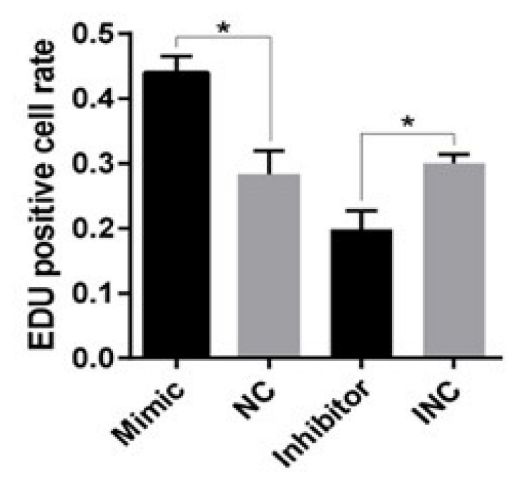

(c)
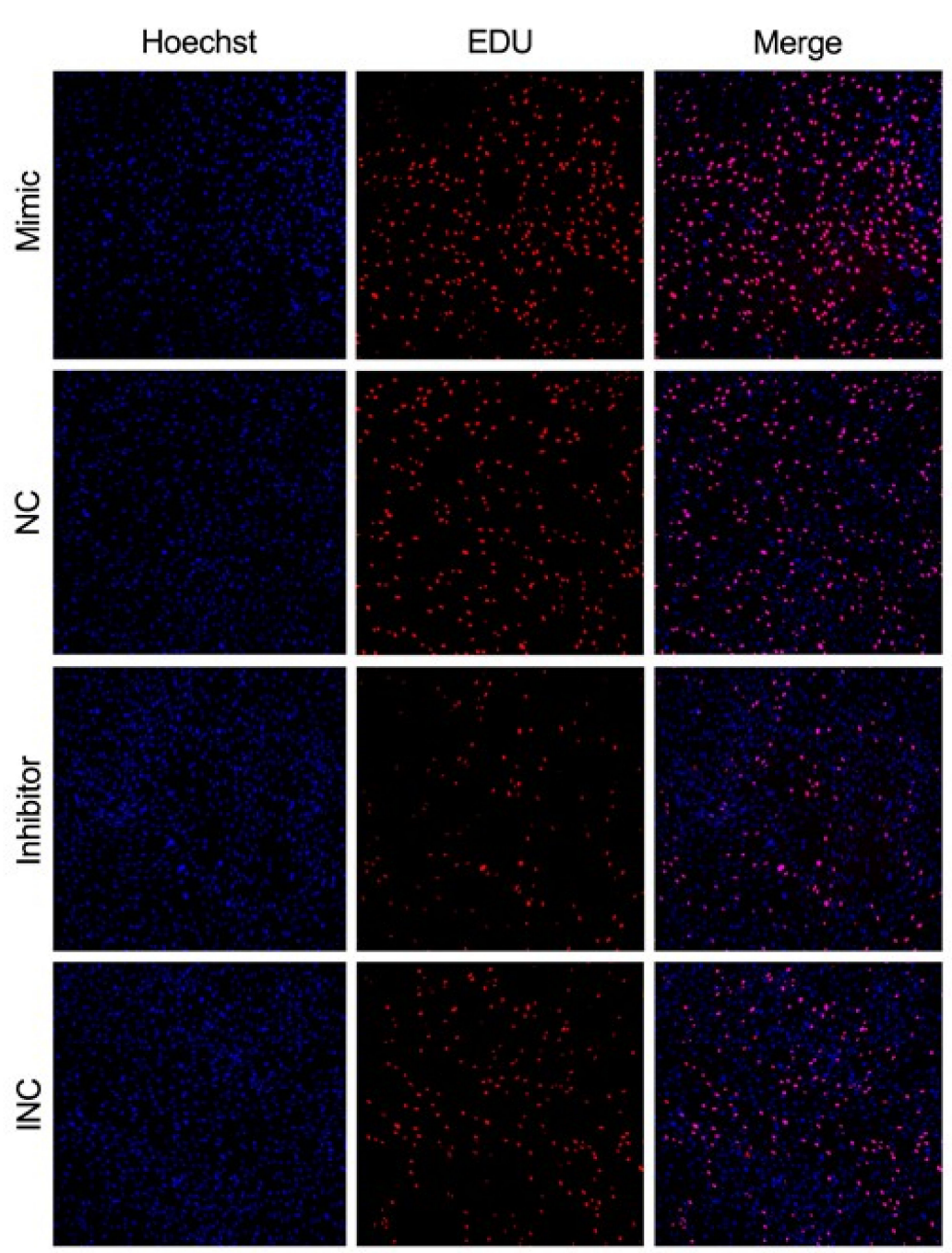

(d)

Figure 3. miR-208b promotes rabbit preadipocyte proliferation. (a,b) The absorbance of preadipocyte at 24, 48, 72, 96, and $120 \mathrm{~h}$ after transfection with the miR-208b mimic, NC, miR-208b inhibitor, and INC $(n=6)$. (c) The percent of EDU positive cells. Red fluorescence represents the EDU positive cells, and blue fluorescence represents the Hoechst stained cells. EDU positive cells rate $=$ EDU positive cells $/$ Hoechst stained cells $\times 100 \%(n=3)$. (d) The picture of the EDU proliferation assay for preadipocyte transfected with the miR-208b mimic, NC, miR-208b inhibitor, and INC (red fluorescence represents EDU positive cells and nuclei are indicated by blue fluorescence). The data are presented as means \pm SEM. ${ }^{*} p<0.05$; ${ }^{* *} p<0.01$.

\subsection{CSNK2A2 Is One of the Target Genes of miR-208b}

To identify target genes that bind with miR-208b, we used online software TargetScan to perform target genes prediction, and results showed that 208 genes are the most likely candidates (Table S1). GO analysis found that a total of 62 enriched GO terms (33 biological processes (BP), 12 cellular components (CC), and 17 molecular functions (MF)), and 34 out of 62 GO terms (54.84\%) were significantly enriched with $p<0.05$ (Table S2). The enriched GO terms mainly included positive regulation of transcription from RNA polymerase II promoter (GO: 0045944), regulation of translational initiation (GO: 0006446), and translation initiation factor activity (GO: 0003743) (Figure 4a). Moreover, target genes were enriched in signaling pathways such as the Wnt signaling pathway, adherens junction, etc. (Table S3; Figure $4 \mathrm{~b}$ ). MiRWalk database revealed that CSNK2A2 contains a potential binding site for miR-208b (Figure 4c). This observation was validated by a qRT-PCR assay and a luciferase reporter assay. CSNK2A2 was significantly down-regulated in the miR-208b mimic 
group but upregulated in the inhibitor group in qRT-PCR analysis (Figure 4d). Moreover, luciferase reporter assay revealed that the luciferase activity was highly suppressed in the group containing the wild-type $3^{\prime}$ UTR of CSNK2A2 mRNA but not significantly changed in the mutant group (Figure 4e). These observations imply that CSNK2A2 is a direct miR-208b target.

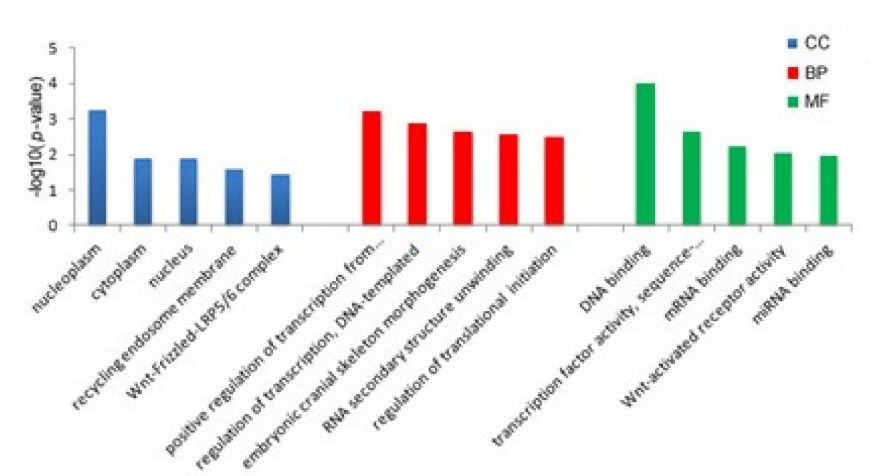

(a)

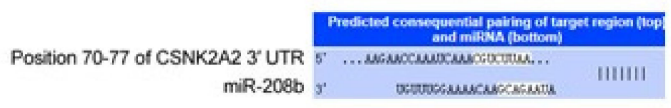

(c)

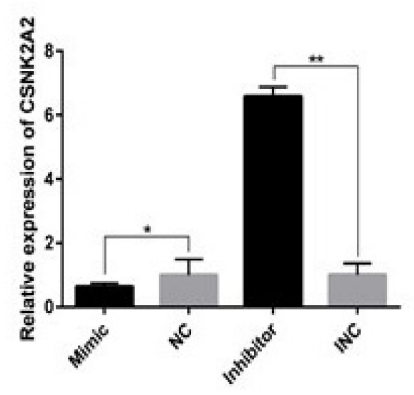

(d)

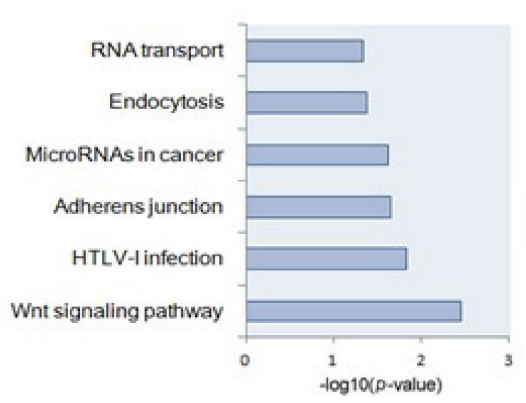

(b)

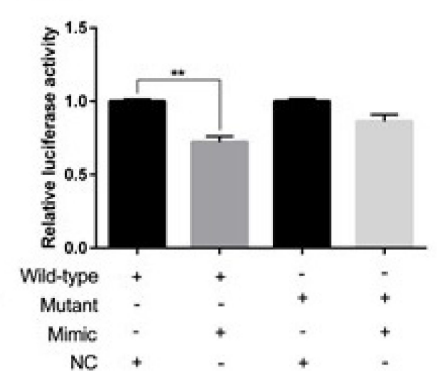

(e)

Figure 4. CSNK2A2 is one of the target genes of miR-208b. (a) GO analysis of miR-208b target genes, only showing the top 5 significantly GO terms in BP, CC, and MF $(p<0.05)$. (b) The significantly enriched pathway of miR-208b target genes by using the KEGG database $(p<0.05)$. (c) The predicted binding site of gene CSNK2A2 with miR-208b. (d) CSNK2A2 mRNA levels after transfecting with the miR-208b mimic, NC, INC, and miR-208b inhibitor $24 \mathrm{~h}$. (e) Luciferase assays were performed by cotransfection of wild-type or mutant plasmids with a miR-208b mimic in Hela cell, and the NC group was used as the control group $(n=3)$. The data are presented as means $\pm \operatorname{SEM}(n=9) .{ }^{*} p<0.05 ;{ }^{* *} p<0.01$.

\section{Discussion}

Currently, obesity has attained the degree of an epidemic, and the prevention and treatment of obesity have been unsuccessful [17]. Adipogenesis is a central event in the process of obesity. Importantly, cell proliferation and differentiation are key processes during adipogenesis and can be regulated by multiple factors [18]. The importance of miRNA was demonstrated in this pathological process $[19,20]$. Recent findings have found that miR-208b is an important factor and responsible for some diseases including chronic chagas disease cardiomyopathy, acute myocardial infarction, and metabolic syndrome $[13,15,21]$. However, the role of miR-208b in preadipocyte proliferation and differentiation has not been carried out. Thus, the detailed information was investigated in preadipocyte to better understand miR-208b-related function.

Hormones and nutrients are necessary for preadipocyte to transform into mature adipocyte capable of lipid synthesis, storage, and production of adipokines [4]. In our study, we applied exogenous drugs DEX, IBMX, and insulin to induce preadipocyte differentiation. $P P A R \gamma$ and $F A B P 4$ are critical transcription factors in the development and function of the fat tissue and markers of preadipocyte differentiation [22,23]. The overexpression of PPAR $\gamma$ in preadipocytes leads to differentiation into mature adipocytes with adipogenic properties and adipokine expression [24]. Moreover, transcription factor $F A B P 4$ is sufficient to induce preadipocyte differentiation and is correlated with markers of 
metabolic syndrome and related disease $[25,26]$. Here, in all tasted in vitro cell samples, qRT-PCR revealed that $P P A R \gamma$ and FABP4 expression were detected and upregulated in the differentiation process, which was consistent with the previous finding [16]. Besides, we also observed different lipid drops by Oil Red $\mathrm{O}$ staining assay, compared with results from the qRT-PCR trial, indicating that preadipocyte was successfully induced to differentiate. Meanwhile, miR-208b level during the differentiation model reveals a possible regulatory role of miR-208b in preadipocyte differentiation.

In humans and animals, white fat tissue is a multifunctional organ supporting triglyceride storage for energy demands. Detailly, intracellular triglyceride is mainly derived from circulating non-esterified fatty acids and/or through hydrolysis of lipoproteins. As the precursor of mature adipocyte, preadipocyte is capable of proliferation and differentiation and these two processes are happened in quick succession [27]. Moreover, the number of adipocytes in white fat tissue mainly depends on the number of preadipocyte, endocrine, and dietary stimuli [28]. Thus, the proliferation and differentiation of preadipocyte are contributed to adipose tissue development and even body weight gain. In the present study, we found that upregulation of miR-208b increased the expression of $P P A R \gamma$ and $F A B P 4$, but downregulation of miR-208b reduced the expression of PPAR $\gamma$ and FABP4 at both mRNA and protein levels. Moreover, the number of lipid droplets was found to be higher after transfection of miR-208b. These results suggest that miR-208b promotes the differentiation of preadipocyte and accumulation of intracellular triglyceride. Moreover, to intuitively determine the functional consequences of miR-208b in the proliferation of preadipocyte, we performed EDU and CCK analysis and found that miR-208b not only positively regulates the differentiation of preadipocyte but also enhance the proliferation of preadipocyte. Interestingly, a previous study revealed that miR-208b accelerates the proliferation and inhibits the differentiation of myogenic cells by targeting TCF12 [29]. Thus, we conclude that miR-208b play different roles in different cells.

miRNAs are endogenous small RNA that play an important gene-regulatory role in animals by pairing to the mRNA of protein-coding genes to direct their post-transcriptional repression [10]. Here, a total of 208 target genes were identified using the online software TargetScan and were further analyzed with GO and KEGG to obtain an overview of these target genes and to further explore the function of miR-208b. GO and KEGG are convenient tools for understanding the biological function of genes and gene products [30]. The significantly enriched GO terms in the BP, CC, and MF revealed the possible function of miR-208b target genes in regulating adipogenesis. For example, regulation of translational initiation (GO: 0006446), transcription coactivator activity (GO: 0003713), translation initiation factor activity (GO: 0003743), and regulation of transcription, DNA-templated (GO: 0006355) were significantly enriched. Adipogenesis is a complex process in which pluripotent mesenchymal stem cells differentiate into mature adipocytes and is controlled by a series of regulators [31,32]. Regulating translation during protein synthesis is important for cell proliferation and differentiation [33]. In our KEGG pathway analysis, five pathways were significantly enriched, including the Wnt signaling pathway, adherens junction, etc. Wnt signaling is a molecular switch that controls adipogenesis. Wnt signaling maintains preadipocytes in an undifferentiated state through inhibition of the adipogenic transcription factors C/EBP $\alpha$ and PPAR $\gamma$ [34]. Overall, the Wnt signaling pathway is a negative regulator in adipogenesis when it is activated [35]. Moreover, a major membrane structure to connect cells is adherens junction (AJ), which comprises cadherin receptors, associated proteins termed catenins and cytoskeletons [36]. Dynamic changes in cell adhesion are needed to resolve and establish new cell contacts during developmental cell movements, tissue renewal, and wound repair [37]. Cytoskeletal remodeling and cell-cell interaction are necessary steps in the transformation of preadipocytes into mature adipocytes [38].

\section{Conclusions}

In conclusion, we applied exogenous drugs DEX, IBMX, and insulin to induce preadipocyte differentiation in vitro. We found that miR-208b was differently expressed 
during the progress of differentiation. Functionally, miR-208b positively promoted the expression of preadipocyte differentiation key genes PPAR $\gamma$ and FABP4 at mRNA and protein levels. Both EDU and CCK analysis suggested that miR-208b was associated with preadipocyte proliferation. Overall, our data reveal that miR-208b play a positive role in preadipocyte differentiation and proliferation and may contribute to the prevention and treatment of obesity. However, there are some shortcomings in this study given various constraints. For example, among 208 putative genes, we only focused on CSNK2A2 because it is a cellular activity regulator that is involved in multiple pathways such as the Wnt signaling pathway and adherens junction. The above two pathways are known pathways that regulate the function of preadipocyte. Moreover, given that cell activity is a complex process associated with genes, proteins, metabolites, etc., the candidate genes are also critical and should be taken seriously. Moreover, we did not use a different database to verify the analysis results, only one database cannot cover every aspect of the information. Thus, the further functional verification of miR-208b and miR-208b-target genes network will be important to consider in the future.

Supplementary Materials: The following are available online at https:/ /www.mdpi.com/article/10 .3390/genes12060890/s1, Table S1: The possible target genes of miR-208b, Table S2: The GO analysis result of miR-208b target genes, Table S3: KEGG pathways analysis of miR-208b target genes.

Author Contributions: Project administration, J.W. and X.J., Funding acquisition, S.L.; Data curation, T.T. and Y.L.; Writing-original draft, J.S. and T.P. All authors have read and agreed to the published version of the manuscript.

Funding: This work was funded by the China Agricultural Research System (Grant No. CARS-44-A-2).

Institutional Review Board Statement: All experiments in the present trial involving animals were performed under the direction of the Institutional Animal Care and Use Committee from the College of Animal Science and Technology, Sichuan Agricultural University, China (DKY-B2019202015).

Informed Consent Statement: Not applicable.

Data Availability Statement: All data generated or analyzed during this study are included.

Conflicts of Interest: The authors declare no conflict of interest.

\section{References}

1. Shao, J.; Bai, X.; Pan, T.; Li, Y.; Jia, X.; Wang, J.; Lai, S. Genome-Wide DNA Methylation Changes of Perirenal Adipose Tissue in Rabbits Fed a High-Fat Diet. Animals 2020, 10, 2213. [CrossRef] [PubMed]

2. Chaput, J.P.; Saunders, T.J. Bioenergetics of Obesity: Is Fat Gain a Problem or a Solution? J. Parenter. Enter. Nutr. 2012, 1, 4111-4122. [CrossRef]

3. Berg, A.H. Adipocyte differentiation induces dynamic changes in NF-kB expression and activity. AJP Endocrinol. Metab. 2004, 287, E1178-E1188. [CrossRef]

4. Stephen, R.F. Transcriptional control of adipocyte formation. Cell Metab. 2006, 4, 263-273.

5. Zhang, X.J.; Chinkes, D.L.; Asle, A.; Herndon, D.N.; Wolfe, R.R. Lipid metabolism in diet-induced obese rabbits is similar to that of obese humans. J. Nutr. 2008, 138, 515-518. [CrossRef] [PubMed]

6. Fan, J.; Kitajima, S.; Watanabe, T.; Xu, J.; Zhang, J.; Liu, E.; Chen, Y.E. Rabbit models for the study of human atherosclerosis: From pathophysiological mechanisms to translational medicine. Pharmacol. Ther. 2015, 146, 104-119. [CrossRef] [PubMed]

7. Zotte, A.D.; Szendro, Z. The role of rabbit meat as functional food. Meat Sci. 2011, 88, 319-331. [CrossRef] [PubMed]

8. Testroet, E.D.; Yoder, C.L.; Testroet, A.L.; Reynolds, C.J.; O’Neil, M.; Lei, S.M.; Beitz, D.C.; Baas, T.J. Relationship of Fat Quality and Meat Quality Traits of Fresh Pork. Iowa State Univ. Digit. Repos. 2015, 661. [CrossRef]

9. Webb, E.C.; O'Neill, H.A. The animal fat paradox and meat quality. Meat Sci. 2008, 80, 28-36. [CrossRef] [PubMed]

10. Bartel, D.P. MicroRNAs: Target recognition and regulatory functions. Cell 2009, 136, 215-233. [CrossRef] [PubMed]

11. Zhang, P.; Du, J.; Wang, L.; Niu, L.; Zhao, Y.; Tang, G.; Jiang, Y.; Shuai, S.; Bai, L.; Li, X.J.B.; et al. MicroRNA-143a-3p modulates preadipocyte proliferation and differentiation by targeting MAPK7. Biomed. Pharmacother. 2018, 108, 531-539. [CrossRef]

12. Xu, Y.; Du, J.; Zhang, P.; Zhao, X.; Li, Q.; Jiang, A.; Jiang, D.; Tang, G.; Jiang, Y.; Wang, J.; et al. MicroRNA-125a-5p mediates 3T3-L1 preadipocyte proliferation and differentiation. Molecules 2018, 23, 317. [CrossRef] [PubMed]

13. Ferreira, L.R.P.; Frade, A.F.; Santos, R.H.B.; Teixeira, P.C.; Cunha-Neto, E. MicroRNAs miR-1, miR-133a, miR-133b, miR-208a and miR-208b are dysregulated in Chronic Chagas disease Cardiomyopathy. Int. J. Cardiol. 2014, 175, 409-417. [CrossRef] [PubMed] 
14. Li, C.; Chen, X.; Huang, J.; Sun, Q.; Wang, L. Clinical impact of circulating miR-26a, miR-191, and miR-208b in plasma of patients with acute myocardial infarction. Eur. J. Med Res. 2015, 20, 58. [CrossRef]

15. Olson, E.S.; van Rooij, E.; Montgomery, R.L.; Grueter, C. Control of Whole Body Energy Homeostasis by microRNA Regulation. U.S. Patent 9,428,749 B2, 30 August 2016.

16. Luo, G.; Hu, S.; Lai, T.; Wang, J.; Lai, S. MiR-9-5p promotes rabbit preadipocyte differentiation by suppressing leptin gene expression. Lipids Health Dis. 2020, 19, 126. [CrossRef]

17. Cordero, P.; Campion, J.; Milagro, F.I.; Martinez, J.A. Transcriptomic and epigenetic changes in early liver steatosis associated to obesity: Effect of dietary methyl donor supplementation. Mol. Genet. Metab. 2013, 110, 388-395. [CrossRef]

18. Rosen, E.D.; Walkey, C.J.; Puigserver, P.; Spiegelman, B.M. Transcriptional regulation of adipogenesis. Genes Dev. 2000, 14, 1293-1307. [PubMed]

19. Takanabe, R.; Ono, K.; Abe, Y.; Takaya, T.; Horie, T.; Wada, H.; Kita, T.; Satoh, N.; Shimatsu, A.; Hasegawa, K. Up-regulated expression of microRNA-143 in association with obesity in adipose tissue of mice fed high-fat diet. Biochem. Biophys. Res. Commun. 2008, 376, 728-732. [CrossRef]

20. Perri, R.; Nares, S.; Zhang, S.; Barros, S.P.; Offenbacher, S. MicroRNA Modulation in Obesity and Periodontitis. J. Dent. Res. 2012, 91, 33-38. [CrossRef]

21. Liu, X.; Yuan, L.; Chen, F.; Zhang, L.; Han, Z. Circulating miR-208b: A Potentially Sensitive and Reliable Biomarker for the Diagnosis and Prognosis of Acute Myocardial Infarction. Clin. Lab. 2017, 63, 101-109. [CrossRef] [PubMed]

22. Siersbæk, R.; Nielsen, R.; Mandrup, S. PPAR $\gamma$ in adipocyte differentiation and metabolism-Novel insights from genome-wide studies. FEBS Lett. 2010, 584, 3242-3249. [CrossRef]

23. Doğan, A.; Demirci, S.; Kıratlı, B.; Şahin, F. Cytoglobin: A potential marker for adipogenic differentiation in preadipocytes in vitro. Cytotechnology 2017, 69, 157-165. [CrossRef]

24. Tontonoz, P.; Hu, E.; Spiegelman, B.M. Stimulation of adipogenesis in fibroblasts by PPAR $\gamma 2$, a lipid-activated transcription factor. Cell 1994, 79, 1147-1156. [CrossRef]

25. Kralisch, S.; Fasshauer, M. Adipocyte fatty acid binding protein: A novel adipokine involved in the pathogenesis of metabolic and vascular disease? Diabetologia 2013, 56, 10-21. [CrossRef] [PubMed]

26. Yang, V.W.; Christy, R.J.; Cook, J.S.; Lane, T.J.K.D. Mechanism of Regulation of the 422(aP2) Gene by cAMP during Preadipocyte Differentiation. Proc. Natl. Acad. Sci. USA 1989, 86, 3629-3633. [CrossRef]

27. Szatkowski, C.; Vallet, J.; Dormishian, M.; Messaddeq, N.; Valet, P.; Boulberdaa, M.; Metzger, D.; Chambon, P.; Nebigil, C.G. Prokineticin Receptor 1 as a Novel Suppressor of Preadipocyte Proliferation and Differentiation to Control Obesity. PLoS ONE 2013, 8, e81175. [CrossRef] [PubMed]

28. Berti, L.; Irmler, M.; Zdichavsky, M.; Meile, T.; Böhm, A. Fibroblast growth factor 21 is elevated in metabolically unhealthy obesity and affects lipid deposition, adipogenesis, and adipokine secretion of human abdominal subcutaneous adipocytes. Mol. Metab. 2015, 4, 519-527. [CrossRef] [PubMed]

29. Fu, L.; Wang, H.; Liao, Y.; Zhou, P.; Li, X. miR-208b modulating skeletal muscle development and energy homeostasis through targeting distinct targets. RNA Biol. 2020, 17, 1728102. [CrossRef] [PubMed]

30. Malhotra, S.; Sowdhamini, R. Sequence search and analysis of gene products containing RNA recognition motifs in the human genome. BMC Genom. 2014, 15, 1159. [CrossRef]

31. Son, Y.H.; Ka, S.; Kim, A.Y.; Kim, J.B. Regulation of Adipocyte Differentiation via MicroRNAs. Endocrinol. Metab. 2014, 29, 122-135. [CrossRef] [PubMed]

32. Koutnikova, H.; Auwerx, J. Regulation of adipocyte differentiation. Ann. Med. 2001, 33, 556-561. [CrossRef] [PubMed]

33. Akabayov, S.R.; Wagner, G. Backbone resonance assignment of the HEAT1-domain of the human eukaryotic translation initiation factor 4GI. Biomol. NMR Assign. 2014, 8, 89-91. [CrossRef]

34. Ross Sarah, E.; Nahid, H.; Longo Kenneth, A.; Bennett Christina, N.; Lucas Peter, C. Inhibition of Adipogenesis by Wnt Signaling. Science 2000, 289, 950-953. [CrossRef]

35. Xi, F.X.; Wei, C.S.; Xu, Y.T.; Ma, L.; He, Y.L.; Shi, X.E.; Yang, G.S.; Yu, T.Y. MicroRNA-214-3p Targeting Ctnnb1 Promotes 3T3-L1 Preadipocyte Differentiation by Interfering with the Wnt/ $\beta$-Catenin Signaling Pathway. Int. J. Mol. Sci. 2019, 20, 1816. [CrossRef] [PubMed]

36. Fuller, M.T.; Davies, E.; Spence, A. PL-04 Regulation of self-renewal, proliferation and differentiation in an adult stem cell lineage. Mech. Dev. 2009, 126, S3. [CrossRef]

37. Harris, T.J.C.; Tepass, U. Adherens junctions: From molecules to morphogenesis. Nat. Rev. Mol. Cell Biol. 2010, 11, 502-514. [CrossRef]

38. Yang, W.; Guo, X.; Thein, S.; Xu, F.; Sugii, S.; Baas, P.W.; Radda, G.K.; Han, W. Regulation of adipogenesis by cytoskeleton remodelling is facilitated by acetyltransferase MEC-17-dependent acetylation of $\alpha$-tubulin. Biochem. J. 2013, 449, 605-612. [CrossRef] [PubMed] 